\title{
Contrast between Lagrangian and Eulerian analytic regularity properties of Euler equations
}

\author{
Peter Constantin ${ }^{\mathrm{a}}$, Igor Kukavica ${ }^{\mathrm{b}}$, Vlad Vicol ${ }^{\mathrm{a}, *}$ \\ a Princeton University, Princeton, NJ 08544, United States \\ ${ }^{\mathrm{b}}$ University of Southern California, Los Angeles, CA 90089, United States
}

Received 3 April 2015; received in revised form 13 July 2015; accepted 26 July 2015

Available online 3 August 2015

\begin{abstract}
We consider the incompressible Euler equations on $\mathbb{R}^{d}$ or $\mathbb{T}^{d}$, where $d \in\{2,3\}$. We prove that:

(a) In Lagrangian coordinates the equations are locally well-posed in spaces with fixed real-analyticity radius (more generally, a fixed Gevrey-class radius).

(b) In Lagrangian coordinates the equations are locally well-posed in highly anisotropic spaces, e.g. Gevrey-class regularity in the label $a_{1}$ and Sobolev regularity in the labels $a_{2}, \ldots, a_{d}$.

(c) In Eulerian coordinates both results (a) and (b) above are false.

(c) 2015 L'Association Publications de l'Institut Henri Poincaré. Published by Elsevier B.V. All rights reserved.
\end{abstract}

MSC: 35Q35; 35Q30; 76D09

Keywords: Euler equations; Lagrangian and Eulerian coordinates; Analyticity; Gevrey class

\section{Introduction}

The Euler equations for ideal incompressible fluids have two formulations, the Eulerian and the Lagrangian one (apparently both due to Euler [9]). In the Eulerian formulation the unknown functions are velocity and pressure, recorded at fixed locations in space. Their time evolution is determined by equating the rates of change of momenta to the forces applied, which in this case are just internal isotropic forces maintaining the incompressible character of the fluid. In the Lagrangian formulation the main unknowns are the particle paths, the trajectories followed by ideal particles labeled by their initial positions. The Eulerian and Lagrangian formulations are equivalent in a smooth regime in which the velocity is in the Hölder class $\mathcal{C}^{s}$, where $s>1$. The particle paths are just the characteristics associated with the Eulerian velocity fields.

\footnotetext{
* Corresponding author.

E-mail addresses: const@math.princeton.edu (P. Constantin), kukavica@usc.edu (I. Kukavica), vvicol@math.princeton.edu (V. Vicol).
} 
In recent years it was proved $[4,12,19,22,13,20,18,10,24,7]$ that the Lagrangian paths are time-analytic, even in the case in which the Eulerian velocities are only $\mathcal{C}^{s}$, with $s>1$. In contrast, if we view the Eulerian solution as a function of time with values in $\mathcal{C}^{s}$, then this function is everywhere discontinuous for generic initial data [5,11,16,17]. This points to a remarkable difference between the Lagrangian and Eulerian behaviors, in the not-too-smooth regime.

In this paper we describe a simple but astonishing difference of behaviors in the analytic regime: The radius of analyticity is locally in time conserved in the Lagrangian formulation (Theorem 1.1), but may deteriorate instantaneously in the Eulerian one (Remark 1.2). Moreover, the Lagrangian formulation allows solvability in anisotropic classes, e.g. functions which have analyticity in one variable, but are not analytic in the others (Theorem 1.5). In contrast, the Eulerian formulation is ill-posed in such functions spaces (Theorem 1.6).

\subsection{Velocity in Lagrangian coordinates}

We consider the Cauchy problem for the incompressible homogeneous Euler equations

$$
\begin{aligned}
u_{t}+u \cdot \nabla u+\nabla p & =0 \\
\nabla \cdot u & =0 \\
u(x, 0) & =u_{0}(x)
\end{aligned}
$$

where $(x, t) \in \mathbb{R}^{d} \times[0, \infty)$, and $d \in\{2,3\}$. In order to state our main results, we first rewrite the Euler equations in Lagrangian coordinates. Define the particle flow map $X$ by

$$
\begin{aligned}
\partial_{t} X(a, t) & =u(X(a, t), t) \\
X(a, 0) & =a
\end{aligned}
$$

where $t \geq 0$, and $a \in \mathbb{R}^{d}$ is the Lagrangian label. The Lagrangian velocity $v$ and the pressure $q$ are obtained by composing with $X$, i.e.,

$$
\begin{aligned}
& v(a, t)=u(X(a, t), t) \\
& q(a, t)=p(X(a, t), t) .
\end{aligned}
$$

The Lagrangian formulation of the Euler equations (1.1)-(1.3) is given in components by

$$
\begin{aligned}
\partial_{t} v^{i}+Y_{i}^{k} \partial_{k} q & =0, \quad i=1, \ldots, d \\
Y_{i}^{k} \partial_{k} v^{i} & =0
\end{aligned}
$$

where we have used the summation convention on repeated indices. The derivatives $\partial_{k}$ are with respect to the label direction $a_{k}$ and $Y_{i}^{k}$ represents the $(k, i)$ entry of the matrix inverse of the Jacobian of the particle map, i.e.,

$$
Y(a, t)=\left(\nabla_{a} X(a, t)\right)^{-1} .
$$

We henceforth drop the index $a$ on gradients, as it will be clear from the context when the gradients are taken with respect to Lagrangian variables $a$ or with respect to the Eulerian variable $x$. From (1.2) it follows that $\operatorname{det}(\nabla X)=1$, and thus, differentiating $\partial_{t} X=v$ with respect to labels, and inverting the resulting matrix, we obtain

$$
Y_{t}=-Y(\nabla v) Y .
$$

The closed system for $(v, q, Y)$ is supplemented with the initial conditions

$$
\begin{aligned}
v(a, 0) & =v_{0}(a)=u_{0}(a) \\
Y(a, 0) & =I
\end{aligned}
$$

where $I$ is the identity matrix. In the smooth category, the Lagrangian equations (1.6)-(1.8) are equivalent to the Eulerian ones (1.1)-(1.3). 


\subsection{Vorticity in Lagrangian coordinates}

For $d=2$ the Eulerian scalar vorticity $\omega=\nabla^{\perp} \cdot u$ is conserved along particle trajectories, that is, the Lagrangian vorticity

$$
\zeta(a, t)=\omega(X(a, t), t)
$$

obeys

$$
\zeta(a, t)=\omega_{0}(a)
$$

for $t \geq 0$. The Lagrangian velocity $v$ may then be computed from the Lagrangian vorticity $\zeta$ using the elliptic curl-div system

$$
\begin{aligned}
\varepsilon_{i j} Y_{i}^{k} \partial_{k} v^{j} & =Y_{1}^{k} \partial_{k} v^{2}-Y_{2}^{k} \partial_{k} v^{1}=\zeta=\omega_{0} \\
Y_{i}^{k} \partial_{k} v^{i} & =Y_{1}^{k} \partial_{k} v^{1}+Y_{2}^{k} \partial_{k} v^{2}=0
\end{aligned}
$$

where $\varepsilon_{i j}$ is the sign of the permutation $(1,2) \mapsto(i, j)$. The equation (1.10) above represents the conservation of the Lagrangian vorticity, while (1.11) stands for the Lagrangian divergence-free condition. Note that the right sides of (1.10)-(1.11) are time-independent.

For $d=3$ the Eulerian vorticity vector $\omega=\nabla \times u$ is not conserved along particle trajectories, and the replacement of (1.9) is the vorticity transport formula

$$
\zeta^{i}(a, t)=\partial_{k} X^{i}(a, t) \omega_{0}^{k}(a) .
$$

Thus, in three dimensions, the elliptic curl-div system becomes

$$
\begin{aligned}
\varepsilon_{i j k} Y_{j}^{l} \partial_{l} v^{k} & =\zeta^{i}=\partial_{k} X^{i} \omega_{0}^{k} \\
Y_{i}^{k} \partial_{k} v^{i} & =0
\end{aligned}
$$

where $\epsilon_{i j k}$ denotes the standard antisymmetric tensor. In order to make use of the identity (1.13), we need to reformulate it so that the right side is time-independent, in analogy to the two-dimensional case. Multiplying (1.13) with $Y_{i}^{m}$ and summing in $i$, we get

$$
\varepsilon_{i j k} Y_{i}^{m} Y_{j}^{l} \partial_{l} v^{k}=\omega_{0}^{m}, \quad m=1,2,3,
$$

which is a form of the Cauchy identity containing only $Y$. Recall here the standard Cauchy invariants $[3,24]$

$$
\varepsilon_{i j k} \partial_{j} v^{l} \partial_{k} X^{l}=\omega_{0}^{i}, \quad i=1,2,3,
$$

which can be obtained by taking the Lagrangian curl of the Weber formula [23,6]. Thus, for $d=3$ we solve (1.14) and (1.15) for $\nabla v$ in terms of $Y$ and $\omega_{0}$. Note that, as in the $d=2$ case, this system has a right side which is time-independent.

\subsection{Isotropic and anisotropic Lagrangian Gevrey spaces}

First we recall the definition of the Gevrey spaces. Fix $r>d / 2$, so that $H^{r}\left(\mathbb{R}^{d}\right.$ ) is an algebra (we may replace $H^{r}\left(\mathbb{R}^{d}\right)$ with $W^{r, p}\left(\mathbb{R}^{d}\right)$ for $r>d / p$ and $p \in(1, \infty)$ ). For a Gevrey-index $s \geq 1$ and Gevrey-radius $\delta>0$, we denote the isotropic Gevrey norm by

$$
\|f\|_{G_{s, \delta}}=\sum_{\beta \geq 0} \frac{\delta^{|\beta|}}{\left.|\beta|\right|^{s}}\left\|\partial^{\beta} f\right\|_{H^{r}}=\sum_{m \geq 0} \frac{\delta^{m}}{m !^{s}} \sum_{|\beta|=m}\left\|\partial^{\beta} f\right\|_{H^{r}}
$$

where $\beta \in \mathbb{N}_{0}^{d}$ is a multi-index. Also, let $G_{s, \delta}$ be the set of functions for which the above norm is finite. When $s=1$ this set consists of analytic functions extendable analytically to the strip of radius $\delta$, and which are bounded uniformly in this strip (the latter property is encoded in the summability property of the norm). 
Similarly, given a coordinate $j \in\{1, \ldots, d\}$, we define the anisotropic $s$-Gevrey norm with radius $\delta>0$ by

$$
\|f\|_{G_{s, \delta}^{(j)}}=\sum_{m \geq 0} \frac{\delta^{m}}{m !^{s}}\left\|\partial_{j}^{m} f\right\|_{H^{r}}
$$

that is, among all multi-indices $\beta$ with $|\beta|=m$, we only consider $\beta=\left(\beta_{k}\right)$ with $\beta_{k}=m \delta_{j k}$, where $\delta_{j k}$ is as usual the Kronecker symbol.

\subsection{Main results}

We have the following statement asserting persistence of the Gevrey radius for solutions of the Lagrangian Euler equation.

Theorem 1.1 (Persistence of the Lagrangian Gevrey radius). Assume that $v_{0} \in L^{2}$ and

$$
\nabla v_{0} \in G_{s, \delta}
$$

for some Gevrey-index $s \geq 1$ and a Gevrey-radius $\delta>0$. Then there exist $T>0$ and a unique solution $(v, Y) \in$ $C\left([0, T] ; H^{r+1}\right) \times C\left([0, T], H^{r}\right)$ of the Lagrangian Euler system (1.6)-(1.8), which moreover satisfies

$$
\nabla v, Y \in L^{\infty}\left([0, T], G_{s, \delta}\right) .
$$

On the other hand, if the uniform analyticity radius of the solution $u(x, t)$ of (1.1)-(1.3) is measured with respect to the Eulerian coordinate $x$, then this radius is in general not conserved in time, as may be seen in the following example.

Remark 1.2 (Decay of the Eulerian analyticity radius). We recall from Remark 1.3 in [15] that there exist solutions to (1.1)-(1.3) whose Eulerian real-analyticity radius decays in time. Consider the explicit shear flow example (cf. [8,2]) given by

$$
u(x, t)=\left(f\left(x_{2}\right), 0, g\left(x_{1}-t f\left(x_{2}\right)\right)\right)
$$

which satisfies (1.1)-(1.2) with vanishing pressure in $d=3$, for smooth $f$ and $g$. For $s=1$ we may for simplicity consider the domain to be the periodic box $[0,2 \pi]^{3}$, and let

$$
f(y)=\sin (y) \quad \text { and } \quad g(y)=\frac{1}{\sinh ^{2}(1)+\sin ^{2}(y)} .
$$

It is easily verified that the uniform in $x_{1}$ and $x_{2}$ real-analyticity radius of $u(x, t)$ decays as

$$
\frac{1}{t+1}
$$

for all $t>0$, and is thus not conserved. Note however that the above example does not provide the necessary counterexample to Theorem 1.1, since $g$ does not belong to the periodic version of $G_{1,1}$. Indeed, $(-1)^{n} g^{(2 n)}(0) \geq(2 n !) / 4$, and thus the series defining $\|g\|_{G_{1,1}}$ is not summable.

The next statement shows indeed that Theorem 1.1 does not hold in the Eulerian setting.

Theorem 1.3. There exists a smooth periodic divergence-free function $u_{0}$ such that

$$
\left\|u_{0}\right\|_{G_{1,1}}<\infty
$$

and such that

$$
\|u(t)\|_{G_{1,1}}=\infty
$$

for any $t>0$. 
The example proving Theorem 1.3 is provided in Section 5.

We are indebted to A. Shnirelman [21] for the following example, pointing out that the results of Theorem 1.1 are sharp in the sense that the time of persistence $T$ of the Lagrangian analyticity radius may be strictly less than the maximal time of existence of a real-analytic solution.

Remark 1.4 (Time of persistence of the Lagrangian analyticity radius). (See [21].) Consider the stationary solution

$$
u\left(x_{1}, x_{2}\right)=\left(\sin x_{1} \cos x_{2},-\cos x_{1} \sin x_{2}\right)
$$

of the Euler equations in $\mathbb{R}^{2}$. This is an entire function of $\left(x_{1}, x_{2}\right)$, and moreover, the $x_{1}$-axis is invariant under the induced dynamics. Abusing notation we denote by

$$
X_{1}\left(a_{1}, t\right)=X_{1}\left(a_{1}, 0, t\right)
$$

the image of the point $\left(a_{1}, 0\right)$ under the flow map at the moment $t$, and by

$$
Y_{22}\left(a_{1}, t\right)=Y_{22}\left(a_{1}, 0, t\right)=\left(\partial_{a_{1}} X_{1}\right)\left(a_{1}, 0, t\right)
$$

its Lagrangian tangential derivative. These functions satisfy the ODE

$$
\begin{aligned}
& \frac{d}{d t} X_{1}\left(a_{1}, t\right)=\sin \left(X_{1}\left(a_{1}, t\right)\right), \quad X_{1}\left(a_{1}, 0\right)=a_{1}, \\
& \frac{d}{d t} Y_{22}\left(a_{1}, t\right)=\cos \left(X_{1}\left(a_{1}, t\right)\right) Y_{22}\left(a_{1}, t\right), \quad Y_{22}\left(a_{1}, 0\right)=1 .
\end{aligned}
$$

The solution $X_{1}$ is given by

$$
\cos X_{1}\left(a_{1}, t\right)=\frac{\left(e^{2 t}+1\right) \cos \left(a_{1}\right)-\left(e^{2 t}-1\right)}{\left(e^{2 t}+1\right)-\left(e^{2 t}-1\right) \cos \left(a_{1}\right)}
$$

and its tangential gradient obeys

$$
Y_{22}\left(a_{1}, t\right)=\frac{2 e^{t}}{\left(e^{2 t}+1\right)-\left(e^{2 t}-1\right) \cos \left(a_{1}\right)} .
$$

Thus, for any fixed $t>0$, the function $Y_{22}\left(a_{1}, t\right)$ has a singularity at the complex point $a_{1}=\Re a_{1}+\Im a_{1}$ (and its conjugate) satisfying

$$
\cos \left(a_{1}\right)=\cos \left(\Re a_{1}+i \Im a_{1}\right)=\frac{e^{2 t}+1}{e^{2 t}-1}
$$

so that

$$
\Re a_{1}=0, \quad \text { and } \quad\left|\Im a_{1}\right|=\ln \left(\frac{e^{t}+1}{e^{t}-1}\right) .
$$

Note however that this singularity obeys

$$
\left|a_{1}\right| \rightarrow \infty \quad \text { as } \quad t \rightarrow 0^{+} .
$$

In summary, at any fixed $t>0$ the function $Y(a, t)$ is not anymore entire with respect to the label $a$. Given any $\delta>0$, we have $\nabla_{a} v_{0}=\nabla_{x} u_{0} \in G_{1, \delta}$, and while $\nabla_{x} u(\cdot, t)=\nabla_{x} u_{0} \in G_{1, \delta}$ for all $t>0$, there exists

$$
T=T(\delta)=\ln \left(\frac{e^{\delta}+1}{e^{\delta}-1}\right)>0
$$

such that $Y(\cdot, t)$, and thus also $\nabla_{a} v(\cdot, t)$, obey

$$
\|Y(\cdot, t)\|_{G_{1, \delta}},\|\nabla v(\cdot, t)\|_{G_{1, \delta}} \rightarrow \infty \quad \text { as } \quad t \rightarrow T(\delta)^{-} .
$$

Thus, the time of analyticity radius persistence $T$ guaranteed by Theorem 1.1 cannot be taken as infinite. Yet, Theorem 1.1 is consistent with $T(\delta) \rightarrow 0$ as $\delta \rightarrow \infty$. 
The proof of Theorem 1.1 may be used to obtain the local existence and the persistence of the radius for anisotropic Gevrey spaces as well.

Theorem 1.5 (Solvability in Lagrangian anisotropic Gevrey spaces). For a fixed direction $j \in\{1, \ldots, d\}$, assume that $v_{0} \in H^{r+1}$ and that

$$
\nabla v_{0} \in G_{s, \delta}^{(j)}
$$

for some index $s \geq 1$ and radius $\delta>0$. Then there exist $T>0$ and a unique solution $(v, Y) \in C\left([0, T], H^{r+1}\right) \times$ $C\left([0, T], H^{r}\right)$ of the Lagrangian Euler system (1.6)-(1.8), which moreover satisfies

$$
\nabla v, Y \in L^{\infty}\left([0, T], G_{s, \delta}^{(j)}\right) .
$$

The above theorem does not hold in the Eulerian coordinates as shown by the next result. The fact that the Eulerian version of the theorem does not hold might not surprise, due to the isotropy and time-reversibility of the Euler equations. On the other hand, the fact that the Lagrangian formulation keeps the memory of initial anisotropy is puzzling.

Theorem 1.6 (Ill-posedness in Eulerian anisotropic real-analytic spaces). There exist $T>0$ and an initial datum $u_{0} \in C^{\infty}\left(\mathbb{R}^{2}\right)$ for which $u_{0}$ and $\omega_{0}$ are real-analytic in $x_{1}$, uniformly with respect to $x_{2}$, such that the unique $C\left([0, T] ; H^{r}\right)$ solution $\omega(t)$ of the Cauchy problem for the Euler equations (1.1)-(1.3) is not real-analytic in $x_{1}$, for any $t \in(0, T]$.

\section{Ill-posedness in Eulerian anisotropic real-analytic spaces}

In this section we prove Theorem 1.6. Here, all the derivatives are taken with respect to the Eulerian variables. The idea of the proof is as follows. We consider an initial vorticity that is supported in a horizontal strip around the $x_{1}$-axis and which is nonzero in a horizontal strip and is very highly concentrated near the origin. We can construct it such that it is real analytic in $x_{1}$, but is obviously not real analytic in $x_{2}$. Given that the vorticity is approximately a point vortex at the origin, the corresponding velocity is approximately a pure rotation. Then for short time, the Euler equations will evolve in such manner that the vorticity is supported in a slightly deformed but rotated strip. The rotation uncovers some of the points that were on the boundary of the original strip, making them points of vanishing vorticity, while covering others. Thus, on a horizontal line parallel with the $x_{1}$-axis, the vorticity instantly acquires an interval on which it must vanish, while it is not identically zero, and hence it cannot possibly continue to be real analytic with respect to $x_{1}$.

In the detailed proof we first construct a function

$$
u\left(x_{1}, x_{2}\right) \in C^{\infty}\left(\mathbb{R}^{2}\right)
$$

such that the following properties hold:

(i) $\operatorname{div} u=0$ on $\mathbb{R}^{2}, \operatorname{curl} u=\omega$,

(ii) $\operatorname{supp} \omega \subseteq\left\{\left(y_{1}, y_{2}\right):-1 \leq y_{2} \leq 1\right\}$

(iii) $u_{2}(1,1)>0$ and $u_{2}(-1,1)<0$

(iv) There exists $\varepsilon \in(0,1 / 2)$ such that

$$
\omega\left(x_{1}, x_{2}\right) \neq 0, \quad\left(x_{1}, x_{2}\right) \in\left\{\left(y_{1}, y_{2}\right):\left|y_{1}-1\right|<\varepsilon, 1-\varepsilon<y_{2}<1\right\}
$$

(v) (tangential analyticity for $u$ ) There exist constants $M_{0}, \delta_{0}>0$ such that

$$
\left|\partial_{1}^{m} u\left(x_{1}, x_{2}\right)\right| \leq \frac{M_{0} m !}{\delta_{0}^{m}}
$$

with

$$
\left|\partial_{1}^{m} \partial_{1} u\left(x_{1}, x_{2}\right)\right| \leq \frac{M_{0} m !}{\delta_{0}^{m}}
$$


and

$$
\left|\partial_{1}^{m} \partial_{2} u\left(x_{1}, x_{2}\right)\right| \leq \frac{M_{0} m !}{\delta_{0}^{m}}
$$

(vi) $\partial^{\alpha} \omega$ converges to 0 exponentially fast and uniformly as $x_{1} \rightarrow \pm \infty$, uniformly in $x_{2}$.

In order to simplify the presentation, we introduce the following notation: If $\omega$ is a function (or a measure) with a sufficient decay at infinity, denote

$$
u(\omega)=\int_{\mathbb{R}^{2}} K(x-y) \omega(y) d y
$$

where

$$
K(x)=\frac{1}{2 \pi}\left(-\frac{x_{2}}{|x|^{2}}, \frac{x_{1}}{|x|^{2}}\right)
$$

denotes the Biot-Savart kernel. Now, choose a test function

$$
\psi \in C_{0}^{\infty}(\mathbb{R})
$$

with values in $[0,1]$ such that $\int \psi=1$ with $\psi(x)>0$ for $x \in(-1,1)$ and $\psi=1$ on $[-1 / 4,1 / 4]$. Consider the sequence of vorticities

$$
\omega^{(k)}\left(x_{1}, x_{2}\right)=c_{0} k^{2} \exp \left(-k^{2}\left(x_{1}^{2}+x_{2}^{2}\right)\right) \psi\left(x_{2}\right)
$$

for $k=1,2, \ldots$, where $c_{0}$ is a normalizing constant such that

$$
\int \omega^{(k)}(x) d x \rightarrow 1 \quad \text { as } k \rightarrow \infty
$$

Denote by

$$
u^{(k)}\left(x_{1}, x_{2}\right)=u\left(\omega^{(k)}\left(x_{1}, x_{2}\right)\right), \quad k=1,2, \ldots
$$

the corresponding velocities. Each individual member of this sequence of velocities satisfies the assumptions (i), (ii), (iv), (v), and (vi). (Note however that the constants in (2.1)-(2.3) depend on $k$.) The construction of a desired vorticity is complete once we show that for $k$ large enough, we have

$$
u_{2}^{(k)}(1,1)>0
$$

and

$$
u_{2}^{(k)}(-1,1)<0
$$

These inequalities for $k$ sufficiently large indeed follow immediately once we observe that the sequence (2.4) is an approximation of identity, i.e., it converges to the Dirac mass $\delta_{0}$, while the velocity

$$
u=u\left(\delta_{0}\right)=\frac{1}{2 \pi}\left(-\frac{x_{2}}{|x|^{2}}, \frac{x_{1}}{|x|^{2}}\right)
$$

corresponding to $\delta_{0}$ satisfies (iii). Thus the construction of a velocity satisfying the properties (i)-(vi) is complete. Denote this velocity by $u_{0}$ and the corresponding vorticity $\omega_{0}=\operatorname{curl} u_{0}$. Now, consider the Euler equation

$$
\omega_{t}+u(\omega) \cdot \nabla \omega=0
$$

with

$$
\omega(0)=\omega_{0}
$$

where, recall, $u(\omega)$ denotes the velocity computed from the vorticity $\omega$ via the Biot-Savart law. By the well-known properties of the Euler equation, the solution is smooth for all $t>0$. By (ii) and (iii) and using the Lagrangian variables 
to solve the Euler equation, there exists $t_{0}>0$ with the following property: For every $t \in\left(0, t_{0}\right)$, there exists a constant $\varepsilon_{1}(t)>0$ such that

$$
\omega\left(x_{1}, x_{2}\right)=0, \quad\left|\left(x_{1}, x_{2}\right)-(-1,1)\right|<\varepsilon_{1}(t)
$$

On the other hand, by (iii) and (iv), we obtain, by possibly reducing $t_{0}$, that for every $t \in\left(0, t_{0}\right)$ there exists a constant $\varepsilon_{2}(t)>0$ such that

$$
\omega\left(x_{1}, x_{2}\right) \neq 0, \quad\left|\left(x_{1}, x_{2}\right)-(1,1)\right|<\varepsilon_{2}(t) .
$$

The properties (2.5) and (2.6) contradict the tangential analyticity of $\omega(t)$ at $x_{2}=1$ for all $t \in\left(0, t_{0}\right)$.

\section{Local solvability in Lagrangian anisotropic Gevrey spaces}

In this section we prove Theorem 1.5. For simplicity of the presentation, we give here the proof for $d=2$. The proof carries over mutatis mutandis to $d=3$, where the only change arises from using (1.15) instead of (1.10). These details may be seen in Section 4, where the well-posedness (by which we mean the existence and uniqueness) in $3 d$ isotropic Gevrey spaces is proven.

Fix $s \geq 1$. Without loss of generality, the direction $j \in\{1,2\}$ may be taken to be $j=1$. Fix $\delta>0$ so that $\nabla v_{0} \in G_{s, \delta}^{(1)}$ with the norm $M$, that is, the quantity

$$
\Omega_{m}=\left\|\partial_{1}^{m} \nabla v_{0}\right\|_{H^{r}}
$$

obeys

$$
\sum_{m \geq 0} \Omega_{m} \frac{\delta^{m}}{m !^{s}} \leq M
$$

Recall that $Y_{0}=I$.

Fix $T>0$, to be chosen further below sufficiently small in terms of $M, s$, and $\delta$. For $m \geq 0$ we define

$$
\begin{aligned}
& V_{m}=V_{m}(T)=\sup _{t \in[0, T]}\left\|\partial_{1}^{m} \nabla v(t)\right\|_{H^{r}}, \\
& Z_{m}=Z_{m}(T)=\sup _{t \in[0, T]} t^{-1 / 2}\left\|\partial_{1}^{m}(Y(t)-I)\right\|_{H^{r}} .
\end{aligned}
$$

Observe that in the norm (3.2) the velocity $v$ does not appear without a gradient. Also, we note that the power $-1 / 2$ of $t$ appearing in (3.3) is arbitrary, in the sense that the proof works with any power in $(-1,0)$.

First we bound $\nabla v$ from the approximate curl-div system (1.10)-(1.11), in terms of $Y$ and $\omega_{0}$. Since $\partial_{1}^{m}$ commutes with curl and div, we may use the Helmholtz decomposition to estimate

$$
\left\|\partial_{1}^{m} \nabla v\right\|_{H^{r}} \leq C\left\|\partial_{1}^{m} \operatorname{curl} v\right\|_{H^{r}}+C\left\|\partial_{1}^{m} \operatorname{div} v\right\|_{H^{r}} .
$$

Further, by appealing to (1.10)-(1.11), the Leibniz rule, and the fact that $H^{r}$ is an algebra, we obtain

$$
\begin{gathered}
\left\|\partial_{1}^{m} \nabla v\right\|_{H^{r}} \leq C\left\|\partial_{1}^{m}\left(\omega_{0}+\varepsilon_{i j}\left(\delta_{i k}-Y_{i}^{k}\right) \partial_{k} v^{j}\right)\right\|_{H^{r}}+C\left\|\partial_{1}^{m}\left(\left(\delta_{i k}-Y_{i}^{k}\right) \partial_{k} v^{i}\right)\right\|_{H^{r}} \\
\leq C\left\|\partial_{1}^{m} \omega_{0}\right\|_{H^{r}}+C\|Y-I\|_{H^{r}}\left\|\partial_{1}^{m} \nabla v\right\|_{H^{r}}+C\left\|\partial_{1}^{m}(Y-I)\right\|_{H^{r}}\|\nabla v\|_{H^{r}} \\
\quad+C \sum_{j=1}^{m-1}\left(\begin{array}{c}
m \\
j
\end{array}\right)\left\|\partial_{1}^{j}(Y-I)\right\|_{H^{r}}\left\|\partial_{1}^{m-j} \nabla v\right\|_{H^{r}}
\end{gathered}
$$

Taking a supremum over $t \in[0, T]$ and using the notation (3.2)-(3.3), we obtain

$$
V_{m} \leq C \Omega_{m}+C T^{1 / 2} Z_{0} V_{m}+C T^{1 / 2} Z_{m} V_{0}+C T^{1 / 2} \sum_{j=1}^{m-1}\left(\begin{array}{c}
m \\
j
\end{array}\right) Z_{j} V_{m-j}
$$

for all $m \in \mathbb{N}$, while for $m=0$ we have 


$$
V_{0} \leq C \Omega_{0}+C T^{1 / 2} Z_{0} V_{0}
$$

Note that we have not used here the evolution equation (1.6) for $v$, and have instead appealed to the Lagrangian vorticity conservation (1.10).

In order to estimate $Z_{m}$, we use the Lagrangian evolution (1.8) in integrated form, and obtain

$$
\begin{aligned}
I-Y(t)= & \int_{0}^{t} Y: \nabla v: Y d \tau \\
= & \int_{0}^{t}(Y-I): \nabla v:(Y-I) d \tau+\int_{0}^{t}(Y-I): \nabla v d \tau \\
& +\int_{0}^{t} \nabla v:(Y-I) d \tau+\int_{0}^{t} \nabla v d \tau
\end{aligned}
$$

for all $t \in[0, T]$. Dividing by $t^{1 / 2}$ and taking a supremum over $t \in[0, T]$ it immediately follows from (3.6) that

$$
Z_{0} \leq C T^{1 / 2}\left(1+T^{1 / 2} Z_{0}\right)^{2} V_{0} .
$$

Differentiating (3.6) $m$ times with respect to the label $a_{1}$, using the Leibniz rule, and the fact that $H^{r}$ is an algebra, we arrive at

$$
\begin{aligned}
& \left\|\partial_{1}^{m}(Y(t)-I)\right\|_{H^{r}} \\
& \leq \sum_{|(j, k)| \leq m} \int_{0}^{t}\left(\begin{array}{c}
m \\
j k
\end{array}\right)\left\|\partial_{1}^{j}(Y-I)\right\|_{H^{r}}\left\|\partial_{1}^{k}(Y-I)\right\|_{H^{r}}\left\|\partial_{1}^{m-j-k} \nabla v\right\|_{H^{r}} d \tau \\
& \quad+2 \sum_{j=0}^{m} \int_{0}^{t}\left(\begin{array}{c}
m \\
j
\end{array}\right)\left\|\partial_{1}^{j}(Y-I)\right\|_{H^{r}}\left\|\partial_{1}^{m-j} \nabla v\right\|_{H^{r}} d \tau+\int_{0}^{t}\left\|\partial_{1}^{m} \nabla v\right\|_{H^{r}} d \tau
\end{aligned}
$$

for all $m \geq 1$. Further, dividing by $t^{1 / 2}$, taking a supremum over $t \in[0, T]$ and using the notation (3.2)-(3.3), we obtain

$$
\begin{aligned}
Z_{m} \leq C T^{3 / 2} \sum_{|(j, k)| \leq m}\left(\begin{array}{c}
m \\
j k
\end{array}\right) Z_{j} Z_{k} V_{m-j-k}+C T \sum_{j=0}^{m}\left(\begin{array}{c}
m \\
j
\end{array}\right) Z_{j} V_{m-j}+C T^{1 / 2} V_{m} \\
\leq C T^{1 / 2}\left(T Z_{0}^{2} V_{m}+T Z_{m} Z_{0} V_{0}+T^{1 / 2} Z_{0} V_{m}+T^{1 / 2} Z_{m} V_{0}+V_{m}\right) \\
\quad+C T^{3 / 2} \sum_{0<|(j, k)|<m}\left(\begin{array}{c}
m \\
j k
\end{array}\right) Z_{j} Z_{k} V_{m-j-k}+C T \sum_{j=1}^{m-1}\left(\begin{array}{c}
m \\
j
\end{array}\right) Z_{j} V_{m-j}
\end{aligned}
$$

for some constant $C>0$.

From (3.5) and (3.7) we obtain that for any $t \in(0, T]$ we have

$$
\begin{aligned}
& V_{0}(t) \leq C_{0} \Omega_{0}+C_{0} t^{1 / 2} Z_{0}(t) V_{0}(t) \\
& Z_{0}(t) \leq C_{0} t^{1 / 2} \sup _{\tau \in[0, t)}\left(V_{0}(\tau)\left(1+t^{1 / 2} Z_{0}(\tau)\right)^{2}\right)
\end{aligned}
$$

for some constant $C_{0}>0$, while the initial data obey

$$
\begin{aligned}
& V_{0}(0)=\left\|\nabla v_{0}\right\|_{H^{r}}=\Omega_{0} \leq M \\
& Z_{0}(0)=0 .
\end{aligned}
$$

Here we used that in view of (3.6), as long as $\nabla v$ and $Y$ are bounded in time, we have $t^{-1 / 2}(Y(t)-I) \approx t^{1 / 2} \rightarrow 0$ as $t \rightarrow 0$. By the continuity in time of $V_{0}(t)$ and $Z_{0}(t)$, it follows that there exists 


$$
T_{1}=T_{1}(M)>0
$$

such that

$$
\begin{aligned}
& \sup _{t \in\left[0, T_{1}\right]} V_{0}(t) \leq 3 C_{0} M \\
& \sup _{t \in\left[0, T_{1}\right]} Z_{0}(t) \leq \frac{1}{2} .
\end{aligned}
$$

This is a time of local existence in $H^{r}\left(\mathbb{R}^{d}\right)$ for $\nabla v$ and $a$.

At this stage, we assume that $T$ obeys

$$
T \leq T_{1}
$$

and we define

$$
B_{m}=V_{m}+Z_{m}=\sup _{t \in[0, T]}\left(V_{m}(t)+Z_{m}(t)\right)
$$

for all $m \geq 0$. By (3.9)-(3.10) we have

$$
B_{0} \leq 3 C_{0} M+\frac{1}{2}
$$

Adding (3.4) and (3.8) we arrive at

$$
\begin{gathered}
B_{m} \leq C_{1} \Omega_{m}+C_{1} T^{1 / 2}\left(1+B_{0}+T^{1 / 2} B_{0}+T B_{0}^{2}\right) B_{m} \\
+C_{1} T^{1 / 2}\left(1+T^{1 / 2}\right) \sum_{0<j<m}\left(\begin{array}{c}
m \\
j
\end{array}\right) B_{j} B_{m-j} \\
+C_{1} T^{3 / 2} \sum_{0<|(j, k)|<m}\left(\begin{array}{c}
m \\
j k
\end{array}\right) B_{j} B_{k} B_{m-j-k}
\end{gathered}
$$

for all $m \geq 1$, for some positive constant $C_{1} \geq 1$. In view of (3.12) we may take

$$
0<T=T\left(B_{0}\right)=T(M)<T_{1}
$$

sufficiently small, such that

$$
C_{1} T^{1 / 2}\left(1+B_{0}+T^{1 / 2} B_{0}+T B_{0}^{2}\right) \leq \frac{1}{2} .
$$

We thus obtain from (3.13) and (3.14) that

$$
\begin{gathered}
B_{m} \leq 2 C_{1} \Omega_{m}+2 C_{1} T^{1 / 2}\left(1+T^{1 / 2}\right) \sum_{0<j<m}\left(\begin{array}{c}
m \\
j
\end{array}\right) B_{j} B_{m-j} \\
+2 C_{1} T^{3 / 2} \sum_{0<|(j, k)|<m}\left(\begin{array}{c}
m \\
j k
\end{array}\right) B_{j} B_{k} B_{m-j-k}
\end{gathered}
$$

for all $m \geq 1$.

Finally, denote

$$
\|(\nabla v, Y-I)\|_{\delta, s, T}=\sum_{m \geq 0} \frac{B_{m} \delta^{m}}{m !^{s}} .
$$

Multiplying (3.15) by $\delta^{m} m !^{-s}$, noting that since $s \geq 1$ we have $\left(\begin{array}{c}m \\ j\end{array}\right)^{1-s} \leq 1$ and $\left(\begin{array}{c}m \\ j k\end{array}\right)^{1-s} \leq 1$, and recalling the initial datum assumption (3.1), we arrive at 


$$
\begin{gathered}
\|(\nabla v, Y-I)\|_{\delta, s, T} \leq 2 C_{1} M+2 C_{1} T^{1 / 2}\left(1+T^{1 / 2}\right) \sum_{m \geq 0} \sum_{0<j<m} \frac{B_{j} \delta^{j}}{j !^{s}} \frac{B_{m-j} \delta^{m-j}}{(m-j) !^{s}} \\
+2 C_{1} T^{3 / 2} \sum_{m \geq 0} \sum_{0<|(j, k)|<m} \frac{B_{j} \delta^{j}}{j !^{s}} \frac{B_{k} \delta^{k}}{k !^{s}} \frac{B_{m-j-k} \delta^{m-j-k}}{(m-j-k) !^{s}} \\
\leq 2 C_{1} M+2 C_{1} T^{1 / 2}\left(1+T^{1 / 2}\right)\|(\nabla v, Y-I)\|_{\delta, s, T}^{2} \\
+2 C_{1} T^{3 / 2}\|(\nabla v, Y-I)\|_{\delta, s, T}^{3} .
\end{gathered}
$$

Here we used the discrete Young inequality $\ell^{1} * \ell^{1} \subset \ell^{1}$. In order to conclude the proof, we note that the initial values are $\nabla v_{0}$ obeying (3.1), and $Y_{0}=I$. Thus, at $T=0$ we have

$$
\|(\nabla v, Y-I)\|_{\delta, s, 0} \leq M
$$

and in view of (3.16), if $T$ is taken sufficiently small so that

$$
8 C_{1}^{2} T^{1 / 2}\left(1+T^{1 / 2}\right) M+32 C_{1}^{3} T^{3 / 2} M^{2} \leq \frac{1}{4}
$$

we arrive at

$$
\|(\nabla v, Y-I)\|_{\delta, s, T} \leq 4 C_{1} M .
$$

In summary, we have proven that there exists $T=T(M)>0$, given by (3.11), (3.14), and (3.18), such that

$$
\begin{aligned}
& \sum_{m \geq 0} \sup _{t \in[0, T]}\left(\left\|\partial_{1}^{m} \nabla v(t)\right\|_{H^{r}}+\frac{\left\|\partial_{1}^{m}(Y(t)-I)\right\|_{H^{r}}}{t^{1 / 2}}\right) \frac{\delta^{m}}{m !^{s}} \\
& \quad \leq C \sum_{m \geq 0}\left\|\partial_{1}^{m} \nabla v_{0}\right\|_{H^{r}} \frac{\delta^{m}}{m !^{s}}=C M
\end{aligned}
$$

for some constant $C>0$. This concludes the proof of the a priori estimates needed to establish Theorem 1.5.

Remark 3.1 (Justification of the a priori estimates). Here we show that by using an approximation argument we may rigorously justify the inequality (3.20). Assume that the initial datum $v_{0}$ is real-analytic (e.g., a mollified approximation of the original datum) and it satisfies the inequality (3.17), i.e.,

$$
\sum_{m=0}^{\infty}\left\|\partial_{1}^{m} \nabla v_{0}\right\|_{H^{r}} \frac{\delta^{m}}{m !^{s}} \leq M
$$

for some $\delta>0$ and $s \geq 1$. Then by $[1,15]$ we know that the solution is real-analytic on $\left[0, T_{1}\right)$, where $T_{1}>0$ (cf. (3.11)) is the time of existence of the solution $v$ in $H^{r+1}$, which under the assumptions of the theorem may be taken independently of the mollification parameter, and in particular it is infinite when $d=2$. Thus $B_{m}(t)<\infty$ for all $t \in\left[0, T_{1}\right)$ and all $m \geq 0$.

Let $m_{0} \geq 0$ be an arbitrary integer, and define $\bar{B}_{m}=B_{m}$ for $m \in\left\{0,1, \ldots, m_{0}\right\}$ and $\bar{B}_{m}=0$ for $m \in\left\{m_{0}+1\right.$, $\left.m_{0}+2, \ldots\right\}$. Similarly, denote by $\bar{\Omega}_{m}$ the same type of truncation corresponding to $\Omega_{m}$, for all integers $m \geq 0$. Then $\bar{B}_{m}$ and $\bar{\Omega}_{m}$ satisfy the same recursion relation (3.13), i.e.,

$$
\begin{array}{r}
\bar{B}_{m} \leq C_{1} \bar{\Omega}_{m}+C_{1} T^{1 / 2}\left(1+\bar{B}_{0}+T^{1 / 2} \bar{B}_{0}+T \bar{B}_{0}^{2}\right) \bar{B}_{m} \\
+C_{1} T^{1 / 2}\left(1+T^{1 / 2}\right) \sum_{0<j<m}\left(\begin{array}{c}
m \\
j
\end{array}\right) \bar{B}_{j} \bar{B}_{m-j} \\
+C_{1} T^{3 / 2} \sum_{0<|(j, k)|<m}\left(\begin{array}{c}
m \\
j k
\end{array}\right) \bar{B}_{j} \bar{B}_{k} \bar{B}_{m-j-k}
\end{array}
$$


for all $m \geq 0$. Denote

$$
\bar{S}_{m_{0}}(t)=\sum_{m=0}^{\infty} \frac{\bar{B}_{m}(t) \delta^{m}}{m !^{s}}=\sum_{m=0}^{m_{0}} \frac{B_{m}(t) \delta^{m}}{m !^{s}} .
$$

Note that $\bar{S}_{m_{0}}$ is a continuous function of time and

$$
\bar{S}_{m_{0}}(0) \leq M
$$

Following the derivation in (3.16), we then obtain

$$
\bar{S}_{m_{0}}(t) \leq 2 C_{1} M+2 C_{1} T^{1 / 2}\left(1+T^{1 / 2}\right) \bar{S}_{m_{0}}^{2}+2 C_{1} T^{3 / 2} \bar{S}_{m_{0}}^{3}
$$

for all $t \geq 0$. By (3.22) and the continuity of $\bar{S}_{m_{0}}(t)$, we get

$$
\bar{S}_{m_{0}}(T) \leq 4 C_{1} M
$$

provided that $T<T_{1}$ is chosen to obey (3.11), (3.14), and (3.18). The bound (3.23) may be rewritten as

$$
\sum_{m=0}^{m_{0}} \frac{B_{m}(t)}{m !^{s}} \delta^{m} \leq 4 C_{1} M
$$

for all $t \in[0, T]$, with $T$ as above. Finally, since $m_{0} \geq 0$ is arbitrary, from the monotone convergence theorem we obtain

$$
\sum_{m=0}^{\infty} \frac{B_{m}(t)}{m !^{s}} \delta^{m} \leq 4 C_{1} M
$$

for all $t \in[0, T]$. Passing to zero in the mollification approximation completes the proof.

\section{Local in time persistence of the Lagrangian Gevrey radius}

In this section we prove Theorem 1.1. For simplicity of the presentation, we give here the proof for $d=3$. Fix $s \geq 1$ and $\delta>0$ so that $\nabla v_{0} \in G_{s, \delta}$ with norm $M$, that is, the quantity

$$
\Omega_{m}:=\sum_{|\alpha|=m}\left\|\partial^{\alpha} \nabla v_{0}\right\|_{H^{r}}
$$

obeys

$$
\sum_{m \geq 0} \Omega_{m} \frac{\delta^{m}}{m !^{s}} \leq M
$$

Fix $T>0$, to be chosen later sufficiently small in terms of $M, s$, and $\delta$. Similarly to the previous section for $m \geq 0$ define

$$
\begin{aligned}
& V_{m}=V_{m}(T)=\sup _{t \in[0, T]} \sum_{|\alpha|=m}\left\|\partial^{\alpha} \nabla v(t)\right\|_{H^{r}}, \\
& Z_{m}=Z_{m}(T)=\sup _{t \in[0, T]} t^{-1 / 2} \sum_{|\alpha|=m}\left\|\partial^{\alpha}(Y(t)-I)\right\|_{H^{r}} .
\end{aligned}
$$

In order to estimate $\nabla v$ and its derivatives, we use the three-dimensional curl-div system (1.14) and (1.15) to write

$$
\begin{aligned}
&(\operatorname{curl} v)^{m}=\varepsilon_{m l k} \partial_{l} v^{k}=\omega_{0}^{m}+\varepsilon_{i l k}\left(\delta_{i m}-Y_{i}^{m}\right) \partial_{l} v^{k}+\varepsilon_{m j k}\left(\delta_{j l}-Y_{j}^{l}\right) \partial_{l} v^{k} \\
&-\varepsilon_{i j k}\left(\delta_{i m}-Y_{i}^{m}\right)\left(\delta_{j l}-Y_{j}^{l}\right) \partial_{l} v^{k} \\
& \operatorname{div} v=\left(\delta_{i k}-Y_{i}^{k}\right) \partial_{k} v^{i} .
\end{aligned}
$$

From (4.4)-(4.5) we conclude that for $\alpha \in \mathbb{N}_{0}^{3}$ we have 


$$
\begin{aligned}
\left\|\partial^{\alpha} \nabla v\right\|_{H^{r}} \leq C & \left\|\partial^{\alpha} \omega_{0}^{m}\right\|_{H^{r}}+C\left\|\partial^{\alpha}\left(\varepsilon_{i j k}\left(\delta_{i m}-Y_{i}^{m}\right)\left(\delta_{j l}-Y_{j}^{l}\right) \partial_{l} v^{k}\right)\right\|_{H^{r}} \\
& +C\left\|\partial^{\alpha}\left(\varepsilon_{m j k}\left(\delta_{j l}-Y_{j}^{l}\right) \partial_{l} v^{k}\right)\right\|_{H^{r}}+C\left\|\partial^{\alpha}\left(\varepsilon_{i j k}\left(\delta_{i m}-Y_{i}^{m}\right) \partial_{j} v^{k}\right)\right\|_{H^{r}} \\
& +C\left\|\partial^{\alpha}\left(\left(\delta_{i k}-Y_{i}^{k}\right) \partial_{k} v^{i}\right)\right\|_{H^{r}} .
\end{aligned}
$$

Summing the above inequality over all multi-indices with $|\alpha|=m$ and taking a supremum over $t \in[0, T]$ we arrive at

$$
\begin{aligned}
V_{m} \leq & C \Omega_{m}+C T Z_{m} Z_{0} V_{0}+C T Z_{0}^{2} V_{m}+C T^{1 / 2} Z_{0} V_{m}+C T^{1 / 2} Z_{m} V_{0} \\
& +C T^{1 / 2} \sum_{0<j<m} \sum_{|\alpha|=m,|\beta|=j, \beta \leq \alpha}\left(\begin{array}{c}
\alpha \\
\beta
\end{array}\right) \sup _{t \in[0, T]}\left(t^{-1 / 2}\left\|\partial^{\beta}(Y-I)\right\|_{H^{r}}\left\|\partial^{\alpha-\beta} \nabla v\right\|_{H^{r}}\right) \\
& +C T \sum_{0<(j, k)<m|\alpha|=m,|\beta|=j, \beta \leq \alpha,|\gamma|=m-j-k, \gamma \leq \alpha-\beta}\left(\begin{array}{c}
\alpha \\
\beta \gamma
\end{array}\right) \\
& \times \sup _{t \in[0, T]}\left(t^{-1 / 2}\left\|\partial^{\beta}(Y-I)\right\|_{H^{r}} t^{-1 / 2}\left\|\partial^{\gamma}(Y-I)\right\|_{H^{r}}\left\|\partial^{\alpha-\beta-\gamma} \nabla v\right\|_{H^{r}}\right) \\
\leq & C \Omega_{m}+C T Z_{m} Z_{0} V_{0}+C T Z_{0}^{2} V_{m}+C T^{1 / 2} Z_{0} V_{m}+C T^{1 / 2} Z_{m} V_{0} \\
& +C T^{1 / 2} \sum_{0<j<m}\left(\begin{array}{c}
m \\
j
\end{array}\right) Z_{j} V_{m-j}+C T \sum_{0<(j, k)<m}\left(\begin{array}{c}
m \\
j k
\end{array}\right) Z_{j} Z_{k} V_{m-j-k},
\end{aligned}
$$

for all $m \geq 1$. In (4.6) we have used that if $\left\{a_{\alpha}\right\},\left\{b_{\alpha}\right\},\left\{c_{\alpha}\right\}$ are non-negative multi-indexed sequences, then

$$
\sum_{|\alpha|=m,|\beta|=j, \beta \leq \alpha}\left(\begin{array}{c}
\alpha \\
\beta
\end{array}\right) a_{\beta} b_{\alpha-\beta} \leq\left(\begin{array}{c}
m \\
j
\end{array}\right)\left(\sum_{|\beta|=j} a_{\beta}\right)\left(\sum_{|\gamma|=m-j} b_{\gamma}\right)
$$

and

$$
\begin{gathered}
\sum_{|\alpha|=m,|\beta|=j, \beta \leq \alpha,|\gamma|=m-j-k, \gamma \leq \alpha-\beta}\left(\begin{array}{c}
\alpha \\
\beta \gamma
\end{array}\right) a_{\beta} b_{\gamma} c_{\alpha-\beta-\gamma} \\
\leq\left(\begin{array}{c}
m \\
j k
\end{array}\right)\left(\sum_{|\beta|=j} a_{\beta}\right)\left(\sum_{|\gamma|=k} b_{\gamma}\right)\left(\sum_{|\alpha|=m-j-k} c_{\alpha}\right) .
\end{gathered}
$$

These inequalities follow e.g. from [14, Lemma 4.2] and [15, Lemma A.1] and the fact that $\left(\begin{array}{l}\alpha \\ \beta\end{array}\right) \leq\left(\begin{array}{l}|\alpha| \\ |\beta|\end{array}\right)$. Indeed, for (4.7) (the proof of (4.8) being analogous), we have by using the substitution $\gamma=\alpha-\beta$

$$
\begin{aligned}
& \sum_{|\alpha|=m,|\beta|=j, \beta \leq \alpha}\left(\begin{array}{l}
\alpha \\
\beta
\end{array}\right) a_{\beta} b_{\alpha-\beta}=\sum_{|\beta|=j} \sum_{|\gamma|=m-j}\left(\begin{array}{c}
\beta+\gamma \\
\beta
\end{array}\right) a_{\beta} b_{\gamma} \\
& \leq\left(\begin{array}{c}
m \\
j
\end{array}\right) \sum_{|\beta|=j} \sum_{|\gamma|=m-j} a_{\beta} b_{\gamma} .
\end{aligned}
$$

Note that when $m=0$, the bound (4.6) reads as

$$
V_{0} \leq C_{0} \Omega_{0}+C_{0} T^{1 / 2}\left(T^{1 / 2} Z_{0}^{2}+Z_{0}\right) V_{0}
$$

for some constant $C_{0}>0$.

As in the two-dimensional case, in order to bound $Z_{m}$ we appeal to the integral formula for $Y(t)-I$, namely (3.6). We apply $\partial^{\alpha}$ to identity (3.6), sum over all multi-indices with $|\alpha|=m$, divide the resulting inequality by $t^{1 / 2}$ and take a supremum over $t \in[0, T]$. By appealing to (4.7) and (4.8), similarly to (3.8) we obtain

$$
\begin{aligned}
Z_{m} \leq C T^{1 / 2}\left(T Z_{0}^{2} V_{m}+T Z_{m} Z_{0} V_{0}+T^{1 / 2} Z_{0} V_{m}+T^{1 / 2} Z_{m} V_{0}+V_{m}\right) \\
+C T^{3 / 2} \sum_{0<|(j, k)|<m}\left(\begin{array}{c}
m \\
j k
\end{array}\right) Z_{j} Z_{k} V_{m-j-k}+C T \sum_{j=1}^{m-1}\left(\begin{array}{c}
m \\
j
\end{array}\right) Z_{j} V_{m-j}
\end{aligned}
$$


when $m \geq 1$, and

$$
Z_{0} \leq C_{0} T^{1 / 2}\left(1+T^{1 / 2} Z_{0}\right)^{2} V_{0}
$$

for $m=0$.

Once the recursive bounds (4.6)-(4.10) and (4.11)-(4.12) have been established, we combine them with the initial datum assumption (4.1), and as in Section 3 obtain that there exists $T=T(M)>0$ such that

$$
\begin{aligned}
& \sum_{\alpha \geq 0} \sup _{t \in[0, T]}\left(\left\|\partial^{\alpha} \nabla v(t)\right\|_{H^{r}}+\frac{\left\|\partial^{\alpha}(Y(t)-I)\right\|_{H^{r}}}{t^{1 / 2}}\right) \frac{\delta^{|\alpha|}}{|\alpha| !^{s}} \\
& \quad \leq C \sum_{\alpha \geq 0}\left\|\partial^{\alpha} \nabla v_{0}\right\|_{H^{r}} \frac{\delta^{|\alpha|}}{|\alpha| !^{s}}=M,
\end{aligned}
$$

for some constant $C>0$. This concludes the proof of the a priori estimates needed to establish Theorem 1.1.

\section{Example of Eulerian ill-posedness in the analytic class $G_{1, \delta}$}

In this section we prove Theorem 1.3. The idea of the proof is similar to the example given earlier in Remark 1.2, but addresses the fact that functions whose holomorphic extension have a simple pole at $\pm i \delta$ do not lie in $G_{1, \delta}$ (a fact encoded in the sum over $m$, as opposed to a supremum over $m$, defining our real-analytic norm, cf. (1.17) and (5.1)). To address this issue we integrate such a real-valued function four times, so that the holomorphic extension to the strip of radius $\delta$ (where $\delta=1$ ) around the real-axis is also a $C^{2}$ function up to the boundary of this strip (cf. (5.5)). The proof then proceeds by cutting off in a Gaussian way at infinity (cf. (5.9)), which is compatible with real-analyticity, and then periodize the resulting function so that we are dealing with a finite energy function (cf. (5.13)). Verifying that the resulting function $\varphi$ yields the necessary counterexample to prove the theorem follows then from a direct but slightly technical calculation.

Let $f, g$ be two $2 \pi$-periodic functions. Recall (cf. [8,2]) that the function defined by

$$
u\left(x_{1}, x_{2}, x_{3}, t\right)=\left(f\left(x_{2}\right), 0, g\left(x_{1}-t f\left(x_{2}\right)\right)\right)
$$

is an exact solution of the Euler equations posed on $\mathbb{T}^{3}$, where $\mathbb{T}=[-\pi, \pi]$ with the initial datum

$$
u_{0}\left(x_{1}, x_{2}, x_{3}\right)=\left(f\left(x_{2}\right), 0, g\left(x_{1}\right)\right) .
$$

Also, for a $2 \pi$-periodic function $\varphi$ and for $\delta>0$ by definition we have that

$$
\|\varphi\|_{G_{1, \delta}}=\sum_{m=0}^{\infty}\left(\sum_{|\alpha|=m}\left\|\partial^{\alpha} \varphi\right\|_{H^{2}\left(\mathbb{T}^{3}\right)}\right) \frac{\delta^{m}}{m !} .
$$

Note that $H^{2}\left(\mathbb{T}^{3}\right) \subset C^{0}\left(\mathbb{T}^{3}\right)$ in view of the Sobolev embedding. Without loss of generality we fix $\delta=1$ throughout this section.

We start with a few considerations on the real line $\mathbb{R}$. For a function $F \in L^{1}(\mathbb{R})$ we normalize the Fourier transform as

$$
\hat{F}(\xi)=\frac{1}{\sqrt{2 \pi}} \int_{\mathbb{R}} F(x) e^{-i x \xi} d x .
$$

Consider the two decaying real-analytic functions

$$
h_{1}(x)=\sqrt{\frac{2}{\pi}} \frac{1}{1+x^{2}}
$$

and

$$
h_{2}(x)=\frac{1}{\sqrt{2}} \exp \left(-\frac{x^{2}}{4}\right) .
$$


These functions have explicit Fourier transforms that are given by

$$
\hat{h}_{1}(\xi)=\exp (-|\xi|)
$$

and

$$
\hat{h}_{2}(\xi)=\exp \left(-|\xi|^{2}\right)
$$

Define

$$
h(x)=h_{1}(x)-\left(1-(-\Delta)^{1 / 2}+\frac{3}{2}(-\Delta)-\frac{7}{6}(-\Delta)^{3 / 2}\right) h_{2}(x)
$$

In view of the above formulae we have that

$$
\hat{h}(\xi)=\exp (-|\xi|)-\left(1-|\xi|+\frac{3}{2}|\xi|^{2}-\frac{7}{6}|\xi|^{3}\right) \exp \left(-|\xi|^{2}\right) .
$$

Note that

$$
\hat{h}(\xi)=\frac{25|\xi|^{4}}{24}+O\left(|\xi|^{5}\right) \quad \text { as } \quad|\xi| \rightarrow 0
$$

and

$$
\hat{h}(\xi)=\exp (-|\xi|)+O\left(\exp \left(-\frac{|\xi|^{2}}{2}\right)\right) \quad \text { as } \quad|\xi| \rightarrow \infty .
$$

Lastly, we define

$$
H(x)=\int_{0}^{x} \int_{0}^{x_{1}} \int_{0}^{x_{2}} \int_{0}^{x_{3}} h\left(x_{4}\right) d x_{4} d x_{3} d x_{2} d x_{1}
$$

so that

$$
\frac{d^{4}}{d x^{4}} H(x)=h(x) .
$$

By taking the Fourier transform of the above equation we arrive at

$$
\hat{H}(\xi)=\frac{\hat{h}(\xi)}{(i \xi)^{4}}=\frac{1}{|\xi|^{4}}\left(\exp (-|\xi|)-\left(1-|\xi|+\frac{3}{2}|\xi|^{2}-\frac{7}{6}|\xi|^{3}\right) \exp \left(-|\xi|^{2}\right)\right)
$$

Clearly,

$$
\sup _{|\xi| \leq 1}|\hat{H}(\xi)|+\sup _{|\xi| \geq 1}\left(|\xi|^{4} \exp (|\xi|)|\hat{H}(\xi)|\right) \leq C_{0}
$$

for some constant $C_{0}>0$. The function $H$ however is not in $L^{1}$ since it grows as $|x| \rightarrow \infty$, and the above computations are formal. To fix this issue, we set

$$
\Phi(x)=\exp \left(-\frac{x^{2}}{2}\right) H(x) .
$$

This function is smooth, and decays as $|x| \rightarrow \infty$. Moreover, in view of (5.7) and using the explicit Fourier transform of the Gaussian, we have

$$
\begin{aligned}
\hat{\Phi}(\xi) & =\int_{\mathbb{R}} \exp \left(-\frac{(\xi-\eta)^{2}}{2}\right) \hat{H}(\eta) d \eta \\
& =\int_{\mathbb{R}} \exp \left(-\frac{(\xi-\eta)^{2}}{2}\right) \frac{1}{|\eta|^{4}}\left(\exp (-|\eta|)-\left(1-|\eta|+\frac{3}{2}|\eta|^{2}-\frac{7}{6}|\eta|^{3}\right) \exp \left(-|\eta|^{2}\right)\right) d \eta .
\end{aligned}
$$


We claim that

$$
\sup _{|\xi| \leq 1}|\hat{\Phi}(\xi)|+\sup _{|\xi| \geq 1}\left(|\xi|^{4} \exp (|\xi|)|\hat{\Phi}(\xi)|\right) \leq C_{1}
$$

for some universal constant $C_{1}>0$. In order to check whether (5.10) holds, we write

$$
\begin{aligned}
|\xi|^{4} \exp (|\xi|) \hat{\Phi}(\xi)=-\int_{\mathbb{R}} \exp \left(-\frac{(\xi-\eta)^{2}}{2}\right) \exp (|\xi|-|\eta|)|\xi|^{4} \\
\times \frac{1-\left(1-|\eta|+\frac{3}{2}|\eta|^{2}-\frac{7}{6}|\eta|^{3}\right) \exp \left(-|\eta|^{2}+|\eta|\right)}{|\eta|^{4}} d \eta,
\end{aligned}
$$

decompose the above integral in the regions

$$
\left\{|\eta| \leq \frac{1}{4}\right\}, \quad\left\{\frac{1}{4} \leq|\eta| \leq|\xi|^{3 / 4}\right\}, \quad\left\{|\xi|^{3 / 4} \leq|\eta| \leq|\xi|\right\}, \quad\{|\eta| \geq|\xi|\}
$$

and use both the decay resulting from the Gaussian factor and the decay coming from (5.8).

A useful observation that shall be needed below is that we have

$$
\left\||\xi|^{k} \exp (-|\xi|)\right\|_{L^{2}(\mathbb{R})}=\frac{\sqrt{(2 k) !}}{2^{k}}
$$

which by Stirling's estimate

$$
(2 \pi)^{1 / 2} n^{n+1 / 2} e^{-n} \leq n ! \leq e n^{n+1 / 2} e^{-n}, \quad n \in \mathbb{N}
$$

yields

$$
\frac{1}{k !}\left\||\xi|^{k} \exp (-|k|)\right\|_{L^{2}(\mathbb{R})} \leq \frac{1}{k^{1 / 4}} .
$$

Now, we proceed to construct a periodic function with a finite $G_{1,1}$ norm. First, we build a $2 \pi$-periodic function $\varphi$ by using the Poisson summation applied to the function $\Phi$. More precisely, let

$$
\varphi(x)=\sum_{m=-\infty}^{\infty} \Phi(x-2 m \pi) .
$$

Clearly $\varphi$ is periodic, and its Fourier series coefficients obey

$$
\hat{\varphi}(k)=\frac{1}{\sqrt{2 \pi}} \hat{\Phi}(k)
$$

for all $k \in \mathbb{Z}$.

Therefore, using estimates (5.10) and (5.12), with the Poisson summation formula, we have that

$$
\begin{aligned}
\|\varphi\|_{G_{1,1}} & =\sum_{n \geq 0}\left\|\frac{d^{n}}{d x^{n}} \varphi\right\|_{H^{2}(\mathbb{T})} \frac{1^{n}}{n !} \\
& \leq C\|\varphi\|_{H^{6}(\mathbb{T})}+C \sum_{n \geq 5}\left(\left\|\frac{d^{n}}{d x^{n}} \varphi\right\|_{L^{2}(\mathbb{T})}+\left\|\frac{d^{n+2}}{d x^{n+2}} \varphi\right\|_{L^{2}(\mathbb{T})}\right) \frac{1}{n !} \\
& \leq C\|\varphi\|_{H^{6}(\mathbb{T})}+C \sum_{n \geq 5}\left(\left\||k|^{n} \hat{\varphi}(k)\right\|_{L^{2}(\mathbb{Z})}+\left\||k|^{n+2} \hat{\varphi}(k)\right\|_{L^{2}(\mathbb{Z})}\right) \frac{1}{n !} \\
& \leq C\|\varphi\|_{H^{6}(\mathbb{T})}+C \sum_{n \geq 5}\left(\left\||\xi|^{n} \hat{\Phi}(\xi)\right\|_{L^{2}(|\xi| \geq 1)}+\left\||\xi|^{n+2} \hat{\Phi}(\xi)\right\|_{L^{2}(|\xi| \geq 1)}\right) \frac{1}{n !}
\end{aligned}
$$

and thus 


$$
\begin{aligned}
\|\varphi\|_{G_{1,1}} \leq & C\|\varphi\|_{H^{6}(\mathbb{T})} \\
& +C \sum_{n \geq 5}\left(\left\||\xi|^{n-4} \exp (-|\xi|)\right\|_{L^{2}(|\xi| \geq 1)}+\left\||\xi|^{n-2} \exp (-|\xi|)\right\|_{L^{2}(|\xi| \geq 1)}\right) \frac{1}{n !} \\
\leq & C\|\varphi\|_{H^{6}(\mathbb{T})}+C \sum_{n \geq 5}\left(\frac{(n-4) !}{(n-4)^{1 / 4}}+\frac{(n-2) !}{(n-2)^{1 / 4}}\right) \frac{1}{n !} \\
\leq & C\|\varphi\|_{H^{6}(\mathbb{T})}+C \sum_{n \geq 5} \frac{1}{n^{9 / 4}} \\
\leq & C_{\varphi}<\infty .
\end{aligned}
$$

Note that $\|\varphi\|_{G_{1, \delta}}=\infty$ for any analyticity radius $\delta>1$, since

$$
\sum_{n \geq 5} \frac{(n-2) !}{(n-2)^{1 / 4}} \frac{\delta^{n}}{n !}=\infty
$$

whenever $\delta>1$, and the estimate in (5.15) may also be turned into lower bounds.

\section{Proof of Theorem 1.3. Consider}

$$
g(x)=\varphi(x)
$$

where $\varphi$ is as given in (5.13), and define

$$
f(x)=\sin x
$$

Since $f$ is entire, we have that $\|f\|_{G_{1, \delta}}<\infty$ for any $\delta>0$. With the definitions of $f$ and $g$ above, it follows from (5.15) that

$$
\left\|u_{0}\right\|_{G_{1,1}}<\infty \text {. }
$$

Note that in view of the periodicity in $x_{1}$ and $x_{2}$, the functions $f\left(x_{2}\right)$ and $g\left(x_{1}\right)$ have finite energy (i.e., $H^{2}\left(\mathbb{T}^{3}\right)$ becomes $H^{2}(\mathbb{T})$, up to a multiplicative constant), and the multi-index summation in (5.1) becomes a simple sum over $n \geq 0$. Thus (1.19) is established.

In order to establish (1.20), we assume, for the sake of obtaining a contradiction, that for some $t \in(0,1 / 10]$ we have $\|u(t)\|_{G_{1,1}}<\infty$. We fix this value of $t \in(0,1 / 10]$ throughout this proof.

Consider the function

$$
\psi\left(x_{1}, x_{2}\right):=\partial_{x_{1}}^{3} u_{3}\left(x_{1}, x_{2}, x_{3}, t\right)=g^{\prime \prime \prime}\left(x_{1}-t f\left(x_{2}\right)\right) .
$$

The inequality $\|u(t)\|_{G_{1,1}}<\infty$ implies

$$
\sum_{\alpha \geq 0}\left\|\partial^{\alpha} \psi\right\|_{H^{2}} \frac{1}{(|\alpha|+3) !}<\infty .
$$

It follows that for any $R \in(0,1)$, the joint in $\left(x_{1}, x_{2}\right)$ power series of $\psi$ at the origin

$$
\psi\left(x_{1}, x_{2}\right)=\sum_{m, n \geq 0} a_{m, n} x_{1}^{m} x_{2}^{n}
$$

converges absolutely in the closed square of side length $R$ at the origin

$$
\mathcal{C}_{R}=\left\{\left(x_{1}, x_{2}\right):\left|x_{1}\right| \leq R,\left|x_{2}\right| \leq R\right\}
$$

and defines a real-analytic function of two variables in this square. Thus, we may consider the complex extension

$$
\psi\left(z_{1}, z_{2}\right)=\psi\left(x_{1}+i y_{1}, x_{2}+i y_{2}\right)=\sum_{m, n \geq 0} a_{m, n} z_{1}^{m} z_{2}^{n}
$$


which converges absolutely when $\left|z_{1}\right| \leq R$ and $\left|z_{2}\right| \leq R$. Fix

$$
R_{t}=1-\frac{3 t}{4}
$$

which clearly belongs to $(0,1)$, and is thus an allowable choice for $R$. Also, fix

$$
x_{1}=0 \quad \text { and } \quad z_{2}=0+i \log 2 .
$$

Since $t \in(0,1 / 10)$, we have $\left|z_{2}\right|=\log 2<R_{t}$, so that by the above consideration,

$$
\lim _{y_{2} \rightarrow-R_{t}^{-}}\left|\psi\left(i y_{2}, i \log 2\right)\right|<\infty .
$$

In order to complete the proof by contradiction, we shall next show that in fact (5.21) is false, and in fact we have

$$
\lim _{y_{2} \rightarrow-R_{t}^{-}}\left|\psi\left(i y_{2}, i \log 2\right)\right|=\infty .
$$

The remainder of this proof is devoted to establishing (5.22).

First observe that $\sin (i \log 2)=3 i / 4$, and thus

$$
\psi\left(i y_{2}, i \log 2\right)=\varphi^{\prime \prime \prime}\left(i\left(y_{2}-3 t / 4\right)\right) .
$$

Next, note that by the definition of $R_{t},(5.20)$, we have

$$
y_{2}-\frac{3 t}{4} \rightarrow-1^{+} \quad \text { as } \quad y_{2} \rightarrow-R_{t}^{-} .
$$

Thus, proving (5.22) amounts to showing that

$$
\lim _{y \rightarrow-1^{+}}\left|\varphi^{\prime \prime \prime}(i y)\right|=\infty
$$

which is what we establish below. In view of (5.9), (5.13), and the Leibniz rule, we have that

$$
\varphi^{\prime \prime \prime}(z)=\Phi^{\prime \prime \prime}(z)+\sum_{m \in \mathbb{Z} \backslash\{0\}} \Phi^{\prime \prime \prime}(z-2 m \pi)
$$

and

$$
\Phi^{\prime \prime \prime}(z)=\exp \left(-\frac{z^{2}}{2}\right)\left(H^{\prime \prime \prime}(z)-3 z H^{\prime \prime}(z)+3\left(z^{2}-1\right) H^{\prime}(z)+z\left(3-z^{2}\right) H(z)\right)
$$

for any complex number $z$ with $|z|<1$. Next, note that by (5.4) and (5.6), we have

$$
H^{(i v)}(z)=h_{1}(z)+\mathcal{E}_{0}(z)
$$

where

$$
\mathcal{E}_{0}(z)=\left(1-(-\Delta)^{1 / 2}+\frac{3}{2}(-\Delta)-\frac{7}{6}(-\Delta)^{3 / 2}\right) h_{2}(z)
$$

is an entire function (since its Fourier coefficients are given by a polynomial times a decaying Gaussian). Moreover, letting $\mathcal{E}_{1}(z)=\int_{0}^{z} \mathcal{E}_{0}\left(w_{1}\right) d w_{1}, \mathcal{E}_{2}(z)=\int_{0}^{z} \int_{0}^{w_{1}} \mathcal{E}_{0}\left(w_{2}\right) d w_{2} d w_{1}, \mathcal{E}_{3}(z)=\int_{0}^{z} \int_{0}^{w_{1}} \int_{0}^{w_{2}} \mathcal{E}_{0}\left(w_{3}\right) d w_{3} d w_{2} d w_{1}$, and $\mathcal{E}_{4}(z)=\int_{0}^{z} \int_{0}^{w_{1}} \int_{0}^{w_{2}} \int_{0}^{w_{3}} \mathcal{E}_{0}\left(w_{4}\right) d w_{4} d w_{3} d w_{2} d w_{1}$, we immediately obtain that

$$
\mathcal{E}(z)=\mathcal{E}_{1}(z)-3 z \mathcal{E}_{2}(z)+3\left(z^{2}-1\right) \mathcal{E}_{3}(z)+z\left(3-z^{2}\right) \mathcal{E}_{4}(z)
$$

is also an entire function. On the other hand, we may explicitly compute the integrals of $h$ as

$$
\begin{aligned}
& \mathcal{H}_{1}(z)=\int_{0}^{z} h_{1}\left(w_{1}\right) d w_{1}=\sqrt{\frac{2}{\pi}} \arctan z \\
& \mathcal{H}_{2}(z)=\int_{0}^{z} \int_{0}^{w_{1}} h_{1}\left(w_{2}\right) d w_{2} d w_{1}=\sqrt{\frac{2}{\pi}}\left(z \arctan z-\frac{1}{2} \log \left(1+z^{2}\right)\right)
\end{aligned}
$$




$$
\begin{aligned}
\mathcal{H}_{3}(z) & =\int_{0}^{z} \int_{0}^{w_{1}} \int_{0}^{w_{2}} h_{1}\left(w_{3}\right) d w_{3} d w_{2} d w_{1}=\frac{1}{2} \sqrt{\frac{2}{\pi}}\left(z+\left(z^{2}-1\right) \arctan z-z \log \left(1+z^{2}\right)\right) \\
\mathcal{H}_{4}(z) & =\int_{0}^{z} \int_{0}^{w_{1}} \int_{0}^{w_{2}} \int_{0}^{w_{3}} h_{1}\left(w_{4}\right) d w_{4} d w_{3} d w_{2} d w_{1} \\
& =\frac{1}{12} \sqrt{\frac{2}{\pi}}\left(5 z^{2}+2 z\left(z^{2}-3\right) \arctan z-\left(3 z^{2}-1\right) \log \left(1+z^{2}\right)\right)
\end{aligned}
$$

which implies that

$$
\begin{aligned}
\mathcal{H}(z):= & \mathcal{H}_{1}(z)-3 z \mathcal{H}_{2}(z)+3\left(z^{2}-1\right) \mathcal{H}_{3}(z)+z\left(3-z^{2}\right) \mathcal{H}_{4}(z) \\
= & \frac{1}{12} \sqrt{\frac{2}{\pi}}\left(z\left(-18+33 z^{2}-5 z^{4}\right)\right. \\
& \left.\quad+2\left(15-45 z^{2}+15 z^{4}-z^{6}\right) \arctan z+z\left(39-28 z^{2}+3 z^{4}\right) \log \left(1+z^{2}\right)\right) .
\end{aligned}
$$

In summary, with the definition of $\mathcal{E}$ in (5.27) and of $\mathcal{H}$ in (5.28), we have that

$$
\Phi^{\prime \prime \prime}(z)=\exp \left(-\frac{z^{2}}{2}\right)(\mathcal{H}(z)+\mathcal{E}(z)) .
$$

Letting $z=i y$, and using that $\arctan (i y)=i \arctan y$, we arrive at

$$
\Phi^{\prime \prime \prime}(i y)=\exp \left(\frac{y^{2}}{2}\right)(\mathcal{H}(i y)+\mathcal{E}(i y)) .
$$

Since $\mathcal{E}$ is an entire function, we have that $\sup _{y \in[-1,0]}|\mathcal{E}(i y)| \leq C<\infty$. Writing

$$
\begin{aligned}
\mathcal{H}(i y)= & i \sqrt{\frac{2}{\pi}} y^{2} \operatorname{arctanh} y+\frac{i}{12} \sqrt{\frac{2}{\pi}} y\left(-18-33 y^{2}-5 y^{4}\right) \\
& +\frac{i}{12} \sqrt{\frac{2}{\pi}}\left(39+28 y^{2}+3 y^{4}\right)\left(2 \operatorname{arctanh} y+y \log \left(1-y^{2}\right)\right) \\
& +\frac{i}{6} \sqrt{\frac{2}{\pi}}\left(-24+11 y^{2}+12 y^{4}+y^{6}\right) \operatorname{arctanh} y
\end{aligned}
$$

and observing $\lim _{y \rightarrow-1^{+}}\left(2 \operatorname{arctanh} y+y \log \left(1-y^{2}\right)\right)=-\log 4$ and $\lim _{y \rightarrow-1^{+}}(y+1) \operatorname{arctanh} y=0$, we arrive at

$$
\lim _{y \rightarrow-1^{+}}|\mathcal{H}(i y)|=\infty
$$

since arctanh has a logarithmic singularity at $y=-1$. Combined with the above, it follows from (5.32) that

$$
\lim _{y \rightarrow-1^{+}}\left|\Phi^{\prime \prime \prime}(i y)\right|=\infty
$$

which in turn shall imply that (5.23) holds.

Indeed, the only remaining part of the proof is to show that

$$
\lim _{y \rightarrow-1^{+}} \sum_{m \in \mathbb{Z} \backslash\{0\}}\left|\Phi^{\prime \prime \prime}(i y-2 m \pi)\right|<\infty .
$$

The above holds since for each $m \neq 0$ we have that

$$
|\mathcal{H}(i y-2 m \pi)|+|\mathcal{E}(i y-2 m \pi)| \leq P(m)
$$

uniformly for $|y| \in[1 / 2,1]$, where $P$ is a polynomial, and since

$$
\left|\exp \left(-\frac{(i y-2 m \pi)^{2}}{2}\right)\right| \leq \exp \left(\frac{1}{2}-2 m^{2} \pi^{2}\right)
$$


which makes the sum over $m \neq 0$ finite. In order to obtain the first bound, we use (5.31) and the formula

$$
\arctan z=\frac{1}{2} i(\log (1-i z)-\log (1+i z))
$$

where the complex domains of the above logarithms are cut on $[0, \infty)$ and $(-\infty, 0]$ respectively.

\section{Conflict of interest statement}

The authors declare that they have no conflict of interest.

\section{Acknowledgements}

The authors are thankful to Alexander Shnirelman for helpful suggestions. The work of P.C. was supported in part by the NSF grants DMS-1209394 and DMS-1265132, I.K. was supported in part by the NSF grant DMS-1311943, while the work of V.V. was supported in part by the NSF grants DMS-1348193, DMS-1514771, and by an Alfred P. Sloan Research Fellowship (Award Number AWD1004444).

\section{References}

[1] C. Bardos, S. Benachour, M. Zerner, Analycité des solutions périodiques de l'équation d'Euler en deux dimensions, C. R. Acad. Sci. Paris Sér. A-B 282 (1976) A995-A998.

[2] C. Bardos, E.S. Titi, Loss of smoothness and energy conserving rough weak solutions for the $3 d$ Euler equations, Discrete Contin. Dyn. Syst., Ser. S 3 (2) (2010) 185-197.

[3] A.L. Cauchy, Sur l'état du fluide à une époque quelconque du mouvement, in: Mémoires extraits des recueils de l'Académie des sciences de l'Institut de France, Théorie de la propagation des ondes à la surface dun fluide pesant dune profondeur indéfinie, Sciences mathématiques et physiques, Tome I, Seconde Partie, 1827.

[4] J.-Y. Chemin, Régularité de la trajectoire des particules d'un fluide parfait incompressible remplissant l'espace, J. Math. Pures Appl. (9) 71 (5) (1992) 407-417.

[5] A. Cheskidov, R. Shvydkoy, Ill-posedness of basic equations of fluid dynamics in Besov spaces, Proc. Am. Math. Soc. 138 (2010) $1059-1067$.

[6] P. Constantin, An Eulerian-Lagrangian approach for incompressible fluids: local theory, J. Am. Math. Soc. 14 (2) (2001) 263-278 (electronic).

[7] P. Constantin, V. Vicol, J. Wu, Analyticity of Lagrangian trajectories for well posed inviscid incompressible fluid models, arXiv:1403.5749, 2014.

[8] R.J. DiPerna, A.J. Majda, Oscillations and concentrations in weak solutions of the incompressible fluid equations, Commun. Math. Phys 108 (4) (1987) 667-689.

[9] L. Euler, Principes généraux du mouvement des fluides, Académie Royale des Sciences et des Belles Lettres de Berlin, Mémoires 11 (1757) 274-315.

[10] U. Frisch, V. Zheligovsky, A very smooth ride in a rough sea, Commun. Math. Phys. 326 (2) (2014) 499-505.

[11] A. Himonas, A. Alexandrou, G. Misiolek, Non-uniform dependence on initial data of solutions to the Euler equations of hydrodynamics, Commun. Math. Phys. 296 (1) (2010) 285-301.

[12] P. Gamblin, Système d'Euler incompressible et régularité microlocale analytique, Ann. Inst. Fourier (Grenoble) 44 (5) (1994) 1449 -1475.

[13] O. Glass, F. Sueur, T. Takahashi, Smoothness of the motion of a rigid body immersed in an incompressible perfect fluid, Ann. Sci. Éc. Norm. Supér. (4) 45 (1) (2012) 1-51.

[14] I. Kukavica, V. Vicol, The domain of analyticity of solutions to the three-dimensional Euler equations in a half space, Discrete Contin. Dyn. Syst. 29 (1) (2011) 285-303.

[15] I. Kukavica, V. Vicol, On the analyticity and Gevrey-class regularity up to the boundary for the Euler equations, Nonlinearity 24 (3) (2011) 765-796.

[16] G. Misiolek, T. Yoneda, Ill-posedness examples for the quasi-geostrophic and the Euler equations, in: Analysis, Geometry and Quantum Field Theory, in: Contemp. Math., vol. 584, Amer. Math. Soc., 2012, pp. 251-258.

[17] G. Misiolek, T. Yoneda, Loss of continuity of the solution map for the Euler equations in $\alpha$-modulation and Hïder spaces, arXiv:1412.4619, 2014.

[18] N. Nadirashvili, On stationary solutions of two-dimensional Euler equation, Arch. Ration. Mech. Anal. 209 (3) (2013) $729-745$.

[19] P. Serfati, Structures holomorphes à faible régularité spatiale en mécanique des fluides, J. Math. Pures Appl. 74 (2) (1995) $95-104$.

[20] A. Shnirelman, On the analyticity of particle trajectories in the ideal incompressible fluid, arXiv:1205.5837, 2012.

[21] A. Shnirelman, Personal communication, 2015.

[22] F. Sueur, Smoothness of the trajectories of ideal fluid particles with Yudovich vorticities in a planar bounded domain, J. Differ. Equ. 251 (12) (2011) 3421-3449.

[23] H.M. Weber, Über eine Transformation der hydrodynamischen Gleichungen, J. Reine Angew. Math. (Crelle) 68 (1868) $286-292$.

[24] V. Zheligovsky, U. Frisch, Time-analyticity of Lagrangian particle trajectories in ideal fluid flow, J. Fluid Mech. 749 (2014) $404-430$. 